\title{
Fast Dense Feature Extraction with CNNs that have Pooling or Striding Layers
}

\author{
Christian Bailer ${ }^{1}$ \\ christian.bailer@dfki.de
}

Tewodros A. Habtegebrial ${ }^{2}$

tewodros_amberbir.habtegebrial@dfki.de

Kiran Varanasi ${ }^{1}$

kiran.varanasi@dfki.de

Didier Stricker ${ }^{1,2}$

didier.stricker@dfki.de

\author{
${ }^{1}$ German Research Center for Artificial \\ Intelligence (DFKI) \\ Kaiserslautern, DE \\ ${ }^{2}$ University of Kaiserslautern \\ Kaiserslautern, DE
}

\begin{abstract}
In recent years, many publications showed that convolutional neural network based features can have a superior performance to engineered features. However, not much effort was taken so far to extract local features efficiently for a whole image. In this paper, we present an approach to compute patch-based local feature descriptors efficiently in presence of pooling and striding layers for whole images at once. Our approach is generic and can be applied to nearly all existing network architectures. This includes networks for all local feature extraction tasks like camera calibration, Patchmatching, optical flow estimation and stereo matching. In addition, our approach can be applied to other patchbased approaches like sliding window object detection and recognition. We complete our paper with a speed benchmark of popular CNN based feature extraction approaches applied on a whole image, with and without our speedup, and example code (for Torch) that shows how an arbitrary $\mathrm{CNN}$ architecture can be easily converted by our approach.
\end{abstract}

\section{Introduction}

While most CNNs are directly executed on complete images, there are also many important tasks that require patch based CNN processing i.e. executing the same CNN several times on neighboring, overlapping patches in an image. Most of these tasks fall into the category of CNN based feature extraction $[\boldsymbol{\nabla}, \mathbf{⿴}]$. This includes tasks like camera calibration, Patch-

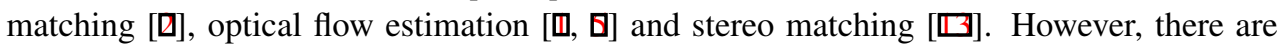
also important patch based applications that are often not considered as feature extraction tasks like sliding window object detection or recognition [0].

In all such patch based tasks there can be a lot of redundancy between the computations of neighboring CNNs, as shown in Figure 1. If there are no pooling or striding layers this redundancy in calculation can easily be avoided by simply executing a CNN which was trained on a limited patch, directly once on the full image. However, with pooling layers the situation is more complex. So far, authors avoided pooling or striding layers completely [ $\square$ ] $]$, 

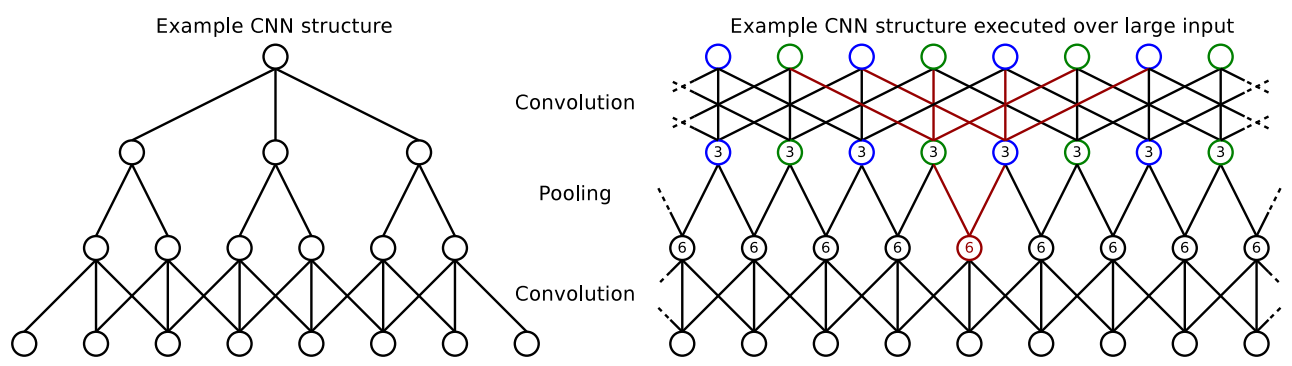

Figure 1: Left: A simple 1 dimensional CNN. Right: if this CNN is executed at each pixel position of an image to create features for every position many intermediate layer results are shared between networks. The numbers in nodes state how often a node is shared. The red connections show how the red node is shared. Pooling with stride 2 halves the output resolution. Thus, we need two pooling layers: the original one (blue) and one shifted by one pixel (green) to avoid halving the output resolution.

simply performed the redundant calculations [ $[$ ], or designed the approach in a way that it can also work with more sparse results $[\mathbf{Q}, \mathbf{\square}]$. The only work that we are aware of, that tries to avoid the redundancy is our previous work [四], where the method for avoiding this redundancy was not detailed.

In this paper, we present an elegant and generalizable solution to avoid this redundancy even in the presence of pooling or striding layers. Our approach requires only layers performing transpose and reshape operations, in addition to ordinary CNN layers. Such operations are available in nearly all machine learning frameworks. Furthermore, our approach can be applied on nearly every existing CNN architecture.

Our paper is structured as follows: after presenting related work in Section 2, we present our approach in Section 3. A benchmark of our approach is performed in Section 4. Finally, in the appendix we present example source code for the deep learning framework Torch to make our contribution even clearer.

\section{Related Work}

Besides extracting robust feature descriptors there was always also the need to compute these features fast. A prominent example for this with engineered features is SURF [ $]$ ]. While its predecessor SIFT [ $[$ ] uses Gaussian filters, SURF uses mean filters. This allows very fast dense feature extraction with integral images at the cost of robustness.

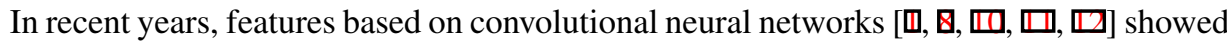
not only promising, but mostly even superior results to engineered features. Zagoruyko and Komodakis [ $\square]$ compared different architectures to compare image patches. While they did not perform a speed comparison they noticed that the Siamese architecture [ $⿴ 囗$ ] with $L_{2}$ distance is much faster than 2-channel based approaches. While the 2-channel architecture requires running a matching CNN for every feature comparison, the Siamese architecture only needs to run a CNN to create a feature but not to match. Once it is created it can be matched by $L_{2}$ distance. Simo-Serra et al. [四] further exploited this idea. However, like [प] they just executed their approach on a predefined set of patches and did not present a fast way to compute their features densely on a whole image. We show in this paper that their 
approach can be adjusted accordingly.

To perform dense stereo matching Zbontar et. al [ $\square]$ ] had to compute features densely on the whole image. They avoided pooling and striding layers by using CNNs only on tiny patches. While CNNs on smaller patches are less robust by themselves, techniques like semi global matching for regularization still allowed them to get state-of-the art results. In contrast, for tasks that cannot be that well regularized like optical flow estimation or image calibration, one has to either follow our approach or do the redundant calculations.

In the application of object recognition the issue of fast dense feature extraction was avoided by simply extracting features sparsely and using regression to find the exact object bounding box [Q] . While this approach is powerful we think that a real dense approach could still improve results, especially in regions where many objects heap or simply to process also less interesting regions with a powerful CNN.

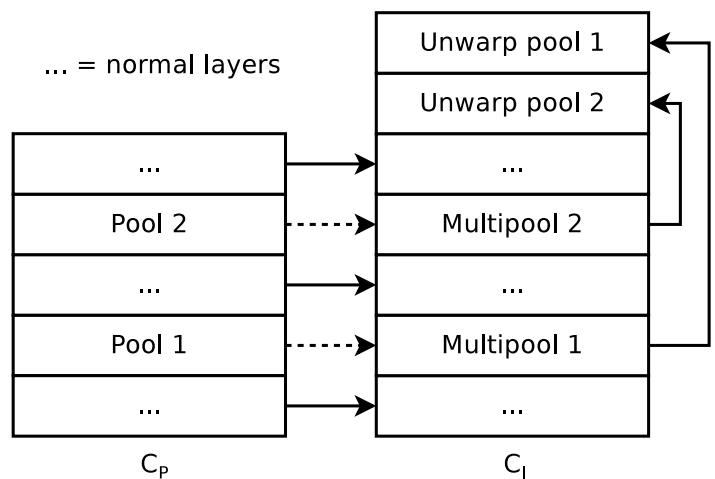

Figure 2: In our approach we create the network $C_{I}$ from network $C_{P} . C_{I}$ gives the same result as executing the network $C_{P}$ on every patch of the image $I$, independently. However, $C_{I}$ runs much faster as it avoids redundancy between overlapping patches.

\section{Approach}

In this section we describe our approach. If we have an input image $I$ with width $I_{w}$ and height $I_{h}$, we can define patches $P(x, y)$ with width $P_{w}$ and height $P_{h}$ centered at each pixel position $(x, y), x \in 0 \ldots I_{w}-1, y \in 0 \ldots I_{h}-1$ in the input image $I$. Patches lying at the image boundary like $P^{I}(0,0)$ require image padding as they contain pixels outside the image area. Still it is common to include such patches to be able to extract features for boundary pixels, as well. Of course it is also possible to only consider patches lying $100 \%$ inside the image area. However, for simplicity we assume that there is a patch surrounding each image pixel.

In this paper we want to efficiently execute a $\mathrm{CNN} C_{P}$ (which was trained on training patches $P^{T}$ ) on all patches $P(x, y)$ in the input image $I$ at once. The output vector $O(x, y)=$ $C_{P}(P(x, y))$ is a $k$ channel vector which belongs to the $\left(I_{h}, I_{w}, k\right)$ dimensional output matrix $O$ that contains the results of $C_{P}$ executed on all image patches $P(x, y)$.

Our goal is to create a network $C_{I}$ that directly calculates $O$ from $I$, while avoiding the redundancy that occurs when $C_{P}$ is executed on each image patch independently. The architectural differences between $C_{P}$ and $C_{I}$ are shown in Figure 2. In the remainder of this sections we describe the steps necessary to get from $C_{P}$ to $C_{I}$, namely: in Section 3.1 we 
describe how to deal with ordinary layers (without pooling or striding). In Section 3.2 we detail how we create multipooling from pooling and finally in Section 3.3 we show how mulipooling can be unwarped again. As notation for single layers of $C_{I}$ and $C_{P}$ we use $L_{I}$ and $L_{P}$, respectively.

\subsection{Layers without pooling}

With no striding or pooling the layers of $C_{P}$ and $C_{I}$ are identical i.e. $L_{P}^{\text {nopool }}=L_{I}^{\text {nopool }}$. This is because their output does not depend on the spacial position of the input, but only on the input values itself.
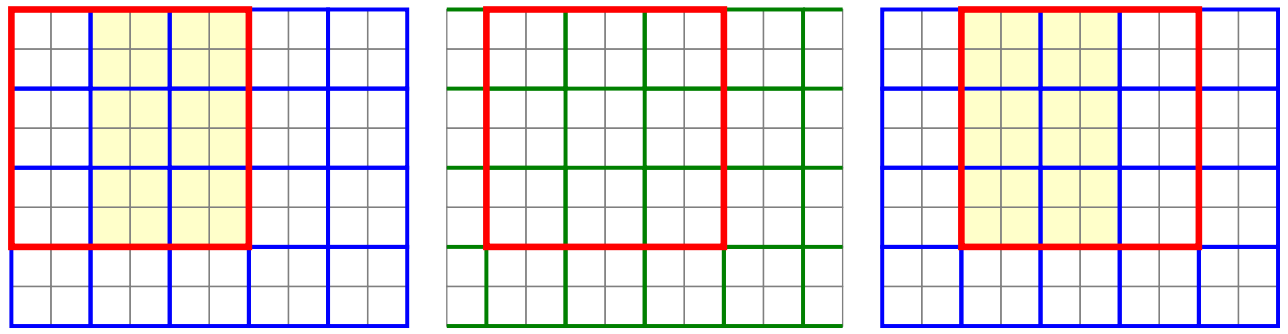

Figure 3: Patches $P$ at different image positions (in red). The first patch $P(x, y)$ requires different $2 \times 2$ pooling (blue) than the second patch $P(x+1, y)$ (green). However, the patch $P(x+2, y)$ can work with the original pooling again (blue). Overlapping positions of $P(x, y)$ and $P(x+2, y)$ provide identical results and can thus be shared (bright yellow). Sharing between patches that are using blue and the ones that are using green pooling is not possible.

\subsection{Multipool to consider all locations}

In contrast to ordinary layers striding and pooling layers must be handled explicitly. However, the kind of pooling has no influence on the handling and striding can be seen as a special kind of pooling layer. Also, it does not make a difference if the pooling layer is executed directly on the input image $I$ or the outputs of one or several preceding layers. If there are preceding layers we simply get a different input $I^{*}$ with patches $P^{*}(x, y)$ that can be processed by the remaining layers $C_{P}^{*}$ or $C_{I}^{*}$, receptively.

Figure 3 visualizes the main issue of pooling: different patches $P(x, y)$ require different poolings even if they are direct neighbors like $P(x, y)$ and $P(x+1, y)$ and can thus not share pooling outputs. However, with $s$ being the pooling/stride size and $u$ and $v$ being integers the patches $P(x, y)$ and $P(x+s u, y+s v)$ still share pooling outputs for pixels that are shared by both patches (yellow area in Figure 3). This creates all together $s \times s$ different pooling situations that have to be computed independently on the input $I^{L}$ of our pooling layer. As a $s \times s$ pooling layer reduces the output size to $I_{w} / s, I_{h} / s$ (with input size $I_{w}, I_{h}$ ) it is clear that $s \times s$ such outputs are required to still obtain an output $O$ of spacial size $I_{w}, I_{h}$.

With $\operatorname{SHIFT}_{y, x}(I)$ being a shifting operation that shifts the input $I$ by $x$ pixels rightwards and $y$ pixels downwards and Pool $_{s \times s}$ being a pooling operation with stride $s \times s$ we can define a shifted pooling operation:

$$
\operatorname{Pool}_{s \times s}^{x, y}(I)=\operatorname{Pool}_{s \times s}\left(\operatorname{SHIFT}_{y, x}(I)\right)
$$


A set of shifted pooling operations with $s \times s$ shift distances we call multipooling. To convert $C_{P}$ to $C_{I}$ we have to replace pooling layers $L_{P}^{\text {pool }} \in C_{P}$ by multipooling layers $L_{I}^{\text {multipool }} \in C_{I}$ :

$$
L_{I}^{\text {multipool }}=\left\{\text { Pool }_{s \times s}^{0,0}, \text { Pool }_{s \times s}^{0,1}, \ldots, \text { Pool }_{s \times s}^{0, s-1}, \ldots, \text { Pool }_{s \times s}^{s-1,0}, \ldots, \text { Pool }_{s \times s}^{s-1, s-1}\right\}
$$

The different pooling outputs are stacked in an extra output dimension which we call $M$. Samples in $M$ are treated as independent samples by subsequent layers (similar to a batch dimension). Note that $M$ actually consists of two dimensions $M=(y, x)$ as the multipooling contains $y$ as well as $x$ shift (and the $y$ shift only increases after processing all $x$ shifts once). If there is more than one pooling layer a subsequent pooling layer replicates the input dimension $M_{\text {in }} s \times s$ times for $M_{\text {out }}$ i.e. $M$ will then consist of $M=\left(y_{n}, x_{n}, \ldots, y_{1}, x_{1}\right)$ after $n$ pooling layers with $y_{1}, x_{1}$ belonging to the first pooling layer and $y_{n}, x_{n}$ to the $n$th pooling layer.

\begin{tabular}{|c|c|c||c|c|c|}
\hline 1,1 & 2,1 & 3,1 & 1,1 & 2,1 & 3,1 \\
\hline 1,2 & 2,2 & 3,2 & 1,2 & 2,2 & 3,2 \\
\hline \hline 1,1 & 2,1 & 3,1 & 1,1 & 2,1 & 3,1 \\
\hline 1,2 & 2,2 & 3,2 & 1,2 & 2,2 & 3,2 \\
\hline
\end{tabular}$\quad \longrightarrow \quad$\begin{tabular}{|l|l|l|l|l|l|l|}
\hline 1,1 & 1,1 & 2,1 & 2,1 & 3,1 & 3,1 \\
\hline 1,1 & 1,1 & 2,1 & 2,1 & 3,1 & 3,1 \\
\hline 1,2 & 1,2 & 2,2 & 2,2 & 3,2 & 3,2 \\
\hline 1,2 & 1,2 & 2,2 & 2,2 & 3,2 & 3,2 \\
\hline
\end{tabular}

Figure 4: Left: $2 \times 2=4$ output images from $2 \times 2$ multipooling. Right: the final unwarping output $O$. We present a generic and efficient way of unwarping in Section 3.3.

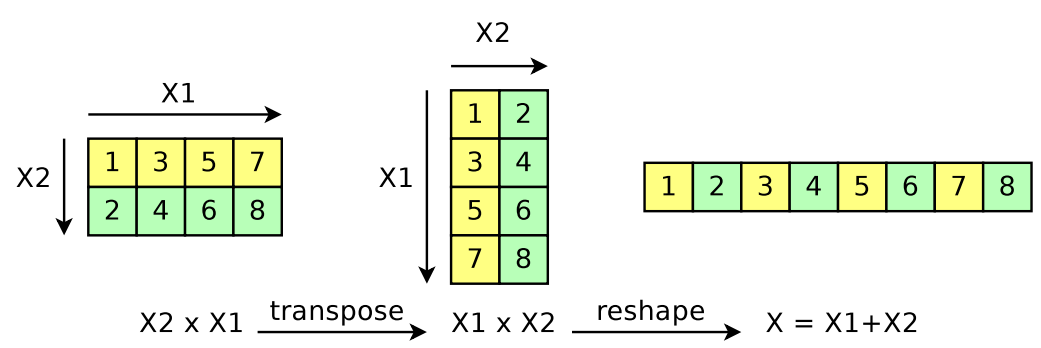

Figure 5: In the problem $\left(x_{2}, x_{1}+1\right)=\left(x_{2}, x_{1}\right)+2$ and $\left(x_{2}, x_{1}\right)=\left(x_{2}, x_{1}\right)+1$ i.e. the step size for the inner dimension $x_{1}$ is larger. This can be fixed by transposing (swapping) both dimensions . A reinterpretation of the memory (reshaping) allows then to reduce it to a single $x$ dimension.

\subsection{Unwarping}

With one multipool layer, we get an output $W$ with dimensions $W=\left(M=s \times s, I_{h} / s, I_{w} / s, k\right)$, which we want to unwarp to the final output $O=\left(I_{h}, I_{w}, k\right)$. Figure 4 shows this unwarping for $2 \times 2$ pooling. Direct unwarping is complex especially with several pooling layers. This might be a reason why previous work avoided pooling layers. However, if we observe the problem in dimension space it can easily be solved with solely transpose and reshape operations. Such operations are supported by most deep learning frameworks as layers.

Let us denote $y^{*}=I_{h} / s, x^{*}=I_{w} / s$ (and $M=\left(y_{1}, x_{1}\right)$ ) for a single pooling layer. Then the dimensions of $W$ can be written as $W=\left(y_{1}, x_{1}, y^{*}, x^{*}, k\right)$. As can be seen in Figure 5 
we have to bring the inner $x$ and $y$ dimensions to the right and the outer ones to the left. The pooling is the inner dimension as it moves pixel by pixel. Thus, $O$ can be written as $O=\left(I_{h}=\left(y^{*}, y_{1}\right), I_{w}=\left(x^{*}, x_{1}\right), k\right) \stackrel{\text { reshape }}{=}\left(y^{*}, y_{1}, x^{*}, x_{1}, k\right)$.

For the more general case of $n$ pooling layers $y^{*}=I_{h} /\left(s_{1} \ldots s_{n}\right), x^{*}=I_{w} /\left(s_{1} \ldots s_{n}\right)$, $W=\left(M=\left(y_{n}, x_{n} \ldots y_{1}, x_{1}\right), y^{*}, x^{*}, k\right)$ we have to get to $O=\left(y^{*}, y_{n}, \ldots, y_{1}, x^{*}, x_{n}, \ldots, x_{1}, k\right)$. An efficient and generic way to do this transformation only with transpose and reshape operations is as follows: first we have to transpose $\left(M, y^{*}, x^{*}, k\right)$ to $\left(y^{*}, x^{*}, M, k\right)$. The naive way requires two transpose operations. However, with reshaping it can be done in one: $\left(M, y^{*}, x^{*}, k\right) \rightarrow\left(M, f^{*}, k\right) \rightarrow\left(f^{*}, M, k\right) \rightarrow\left(y^{*}, x^{*}, M, k\right)$. Note that reshaping requires barely any runtime as it is just a reinterpretation of the memory. Then, we do the following:

1. start: $\left(y^{*}, x^{*}, M, k\right)$

2. reshape $M$ to its contents : $\left(y^{*}, x^{*}, y_{n}, x_{n} \ldots y_{1}, x_{1}, k\right)$

3. do $n$ times

4. transpose: $(\operatorname{dim} 2, \operatorname{dim} 3)$

5. reshape: fuse $(\operatorname{dim} 1, \operatorname{dim} 2)$, fuse $(\operatorname{dim} 3, \operatorname{dim} 4)$

6. end

After performing this algorithm we have determined $O$.

\subsection{Use in practice at the example of torch}

In practice things can differ from theory. In Torch, ${ }^{1}$ for instance, the channel dimension is before the spatial dimension i.e. $W=\left(M, k, y^{*}, x^{*}\right)$. Still we can do our initial step with only one transpose operation: $\left(M, k, y^{*}, x^{*}\right) \rightarrow\left(M, f^{*}\right) \rightarrow\left(f^{*}, M\right) \rightarrow\left(k, y^{*}, x^{*}, M\right)$. To obtain a dimension for $M$ we use an unsqeeze layer before the first CNN layer. An example torch implementation can be found in the appendix.

\section{Experiments}

In this section we present benchmark results of our improved network architecture $C_{I}$ compared to $C_{P}$ running on all patches of an image. The experiments are performed on a GeForce GTX TITAN X. Readers who want to replicate our modify our experiments can use our benchmark code provided in the supplementary material. We do not count the time to extract patches from the image for $C_{P}$, but only the pure network processing time, although including this preprocessing step only required for $C_{P}$ would increase our speedup even more. As can be seen in Table 1, the execution time of $C_{P}$ roughly scales (as expected) linearly with the image pixels. $C_{I}$ on the other hand barely takes more time for larger images. We think that this is due to overhead and because the GPU cores are not fully occupied by $C_{I}$. In theory it should for large images also scale linearly with image pixels. On the other hand, the memory consumption of $C_{I}$ increases nearly linearly. If not enough memory is available the input image can be split into parts and each part can be processed individually. This requires some - in practice barley relevant - computational redundancy. The memory requirement of $C_{P}$ depends on the number of patches processed in parallel. Processing more patches is 


\begin{tabular}{|c|c|c|c|c|c|}
\hline Architecture & Image Size & $C_{P}$ & $C_{I}$ & Speedup & memory $C_{I}$ \\
\hline Simo-Serra et al. [四] & $72 \times 48$ & $1.25 \mathrm{~s}$ & $0.099 \mathrm{~s}$ & 12.6 times & $348 \mathrm{MB}$ \\
\hline Simo-Serra et al. [四] & $360 \times 240$ & $28.42 \mathrm{~s}$ & $0.103 \mathrm{~s}$ & 275 times & $1142 \mathrm{MB}$ \\
\hline Simo-Serra et al. [四] & $720 \times 480$ & $112.76 \mathrm{~s}$ & $0.116 \mathrm{~s}$ & 972 times & $3448 \mathrm{MB}$ \\
\hline Simo-Serra et al. [四] & $1080 \times 720$ & $252.97 \mathrm{~s}$ & $0.117 \mathrm{~s}$ & 1550 times & $7222 \mathrm{MB}$ \\
\hline Zagoruyko et al.[ద]](Siamese $L_{2}$ ) & $1080 \times 720$ & $223.55 \mathrm{~s}$ & $0.127 \mathrm{~s}$ & 1760 times & $11495 \mathrm{MB}$ \\
\hline Bailer et al.[四 (fast arch) & $864 \times 576$ & $363.45 \mathrm{~s}$ & $0.113 \mathrm{~s}$ & 3216 times & $9850 \mathrm{MB}$ \\
\hline
\end{tabular}

Table 1: Speed benchmark for $C_{P}$ and $C_{I}$. The latter performs much faster especially on larger images. We provide source code for the benchmarks in our supplementary material to prove reproducibility. For $C_{P}$ memory is not a big deal (see text).

usually faster, but very large amounts do not have much influence anymore, as GPU cores are limited (still we used large amounts for the fastest possible runtime).

As can also be seen in the table, architectures like [ $\square$ ] that perform heavy convolution can be speed up a little more than architectures that perform heavy pooling like [四, 四].

\section{Conclusion}

In this paper, we presented a novel approach to convert nearly arbitrary CNN architectures for fast execution on the whole image in a sliding window manner. We showed that with our approach significant speedups can be achieved - dense feature maps of state-of-the-art $\mathrm{CNN}$ based feature descriptors can be created in barely more than $0.1 \mathrm{~s}$ for a whole image. This is very interesting considering that $\mathrm{CNN}$ based features are nowadays more robust than most computationally intensive traditional features - and with our approach, it is now also possible to compute them very fast densely.

By providing a straightforward implementation, we hope to convince authors of future works that there is no need to perform unnecessary, redundant calculations or to avoid pooling layers. This is particularly relevant for dense-feature computations on the whole image.

\section{Appendix: Torch example}

Here we present some functions that allow to convert an arbitrary $\mathrm{CNN} C_{P}$ to an architecture $C_{I}$. An example implementation of [四] shows the usage of these functions. Note: [四] uses mean-pooling, but for simplicity we use the much more popular max-pooling in our example. Using mean pooling instead is straightforward.

Torch functionality of our approach:

- Adds padding to the image and adds the dimesion $M$ function multiPoolPrepare (patchY, patchX)

$\operatorname{pad} x=\operatorname{patch} X-1$

pady $=$ patchY -1

local net $=$ nn. Sequential ()

net $: \operatorname{add}(n n \cdot$ Padding $(2,-\operatorname{torch} \cdot \operatorname{ceil}(\operatorname{pady} / 2)))$

net $: \operatorname{add}(n n$. Padding $(3,-\operatorname{torch} \cdot \operatorname{ceil}(\operatorname{padx} / 2)))$

net $: \operatorname{add}(n n$. Padding $(2, \operatorname{torch}$. floor $(\operatorname{pady} / 2)))$ 
net : add (nn. Padding $(3$, torch . floor $(\operatorname{padx} / 2)))$

net : add (nn. Unsqueeze (1))

return net

end

-- Layer prepares unwarping. Added after the network

function unwarpPrepare ()

local net $=$ nn. Sequential ()

net: add (nn. View (-1): setNumInputDims (3)) --puts dim 2,3,4 to one $\operatorname{dim}$ net : add $(n n$. Transpose $(\{1,2\}))$

return net

end

- The actual unwarping. See example for usage

- curImg $=$ imgSize $/($ all still existing strides $)$

function unwarpPool (outChans, curImgW, curImgH, dW, dH)

local net $=$ nn. Sequential ()

net : add (nn. View (outChans, curImgH, curImgW, dH, dW, -1))

net : add $(\mathrm{nn}$. Transpose $(\{3,4\}))--\{3,4\}$ not $\{2,3\}$ as $\mathrm{k}$ is first $\operatorname{dim}$ return net

end

-- Replaces normal maxpooling

function multiMaxPooling $(\mathrm{kW}, \mathrm{kH}, \mathrm{dW}, \mathrm{dH})$

local c1 = nn. DepthConcat (1)

for $\mathrm{i}=0, \mathrm{dH}-1 \mathrm{do}$

for $\mathrm{j}=0, \mathrm{dW}-1 \mathrm{do}$

c1 : add (nn. SpatialMaxPooling $(\mathrm{kW}, \mathrm{kH}, \mathrm{dW}, \mathrm{dH},-\mathrm{j},-\mathrm{i}))$

end

end

return c1

end

Examaple usage:

sL1 $=2-$ stride 1 . pooling layer

sL2 $=3-$ stride 2. pooling layer

sL3 $=4-$ stride 3 . pooling layer

-- image height and width should be multiples of $\mathrm{sL} 1 * \mathrm{sL} 2 * \mathrm{sL} 3$

-- if this is not the case right/downwards padding should be added.

$\mathrm{imH}=\ldots-$ image height

$\mathrm{imW}=\ldots-$ image width

$\mathrm{pH}=64-$ patch height

$\mathrm{pW}=64-\mathrm{patch}$ width

outChans $=128-$ output channels (last layer)

-- The patch network (C_P):

net $1=$ nn. Sequential () 


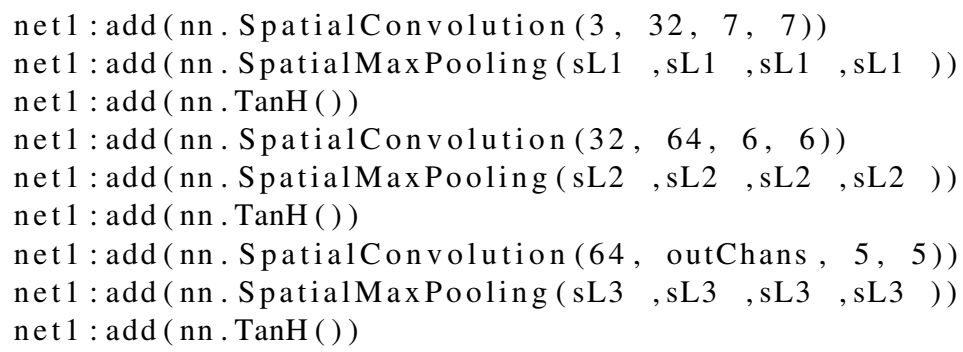

- The image network (C_I):

net $2=$ nn. Sequential ()

net 2 : add (multiPoolPrepare $(\mathrm{pH}, \mathrm{pW}))$

net $2:$ add (net 1 . modules [1])

net 2 : add (multiMaxPooling (sL1, sL1, sL1, sL1))

net 2 : add (net 1 . modules [3])

net 2 : add (net 1 . modules [4])

net 2 : add (multiMaxPooling (sL2, sL2, sL2, sL2 ))

net 2 : add $($ net 1. modules $[6])$

net 2 : add (net 1 . modules [7])

net 2 : add (multiMaxPooling (sL3, sL3, sL3, sL3 ))

net 2 : add ( unwarpPrepare ())

net 2 : add ( unwarpPool (outChans, imH/( sL1*sL2*sL3), imW/( sL1*sL2*sL3), sL3, sL3))

net 2 : add ( unwarpPool (outChans, imH/( sL1*sL2), imW/(sL1*sL2), sL2, sL2))

net 2 : add (unwarpPool (outChans, imH/sL1, imW/sL1 , sL1, sL1) )

net 2 : add $($ nn . View $(-1$, imH , imW $)$

\section{References}

[1] Christian Bailer, Kiran Varanasi, and Didier Stricker. Cnn-based patch matching for optical flow with thresholded hinge loss. arXiv preprint arXiv:1607.08064, 2016.

[2] Connelly Barnes, Eli Shechtman, Dan B Goldman, and Adam Finkelstein. The generalized patchmatch correspondence algorithm. In ECCV, pages 29-43. Springer, 2010.

[3] Herbert Bay, Tinne Tuytelaars, and Luc Van Gool. Surf: Speeded up robust features. Computer vision-ECCV 2006, pages 404-417, 2006.

[4] Jane Bromley, James W Bentz, Léon Bottou, Isabelle Guyon, Yann LeCun, Cliff Moore, Eduard Säckinger, and Roopak Shah. Signature verification using a siamese time delay neural network. International Journal of Pattern Recognition and Artificial Intelligence, 7(04):669-688, 1993.

[5] David Gadot and Lior Wolf. Patchbatch: a batch augmented loss for optical flow. In Proceedings of the IEEE Conference on Computer Vision and Pattern Recognition, pages 4236-4245, 2016. 
[6] Zong Yuan Ge, Chris McCool, Conrad Sanderson, and Peter Corke. Modelling local deep convolutional neural network features to improve fine-grained image classification. In Image Processing (ICIP), 2015 IEEE International Conference on, pages 4112-4116. IEEE, 2015.

[7] Ross Girshick. Fast r-cnn. In Proceedings of the IEEE International Conference on Computer Vision, pages 1440-1448, 2015.

[8] Xufeng Han, Thomas Leung, Yangqing Jia, Rahul Sukthankar, and Alexander C Berg. Matchnet: unifying feature and metric learning for patch-based matching. In Computer Vision and Pattern Recognition (CVPR), 2015.

[9] David G Lowe. Object recognition from local scale-invariant features. In International Conference on Computer Vision (ICCV), 1999.

[10] Edgar Simo-Serra, Eduard Trulls, Luis Ferraz, Iasonas Kokkinos, Pascal Fua, and Francesc Moreno-Noguer. Discriminative learning of deep convolutional feature point descriptors. In International Conference on Computer Vision (ICCV), 2015.

[11] Karen Simonyan, Andrea Vedaldi, and Andrew Zisserman. Learning local feature descriptors using convex optimisation. Pattern Analysis and Machine Intelligence (PAMI), 36(8):1573-1585, 2014.

[12] Sergey Zagoruyko and Nikos Komodakis. Learning to compare image patches via convolutional neural networks. In Computer Vision and Pattern Recognition (CVPR), 2015.

[13] Jure Zbontar and Yann LeCun. Stereo matching by training a convolutional neural network to compare image patches. Journal of Machine Learning Research, 17(1-32): 2, 2016. 